\title{
Dynamic coding of face information in inferior temporal cortex
}

\author{
Yuichiro Yamada', Yoshiki Kashimori ${ }^{1,2^{*}}$ \\ From Nineteenth Annual Computational Neuroscience Meeting: CNS*2010 \\ San Antonio, TX, USA. 24-30 July 2010
}

We can recognize rapidly and effortlessly complex visual scenes. Such amazing ability in visual recognition needs the effective processing of visual information along the multiple stages of visual pathways. Neurophysiological experiments have provided evidence for a "simple-to-complex" processing model based on a hierarchy of increasing complex image features. It has been also shown that inferior temporal (IT) cortex is shape-selective [1,2]. However, little is known about how the information about a whole object and its parts are represented in IT cortex.

Recent experiments have reported dynamic responses of IT neurons in face perception. Hirabayashi and Miyashita [3] demonstrated that the IT neurons exhibited different responses in spike synchronicity for face and non-face images. Sugase et al. [4] found that single IT neurons conveyed two different scales of facial information in their firing patterns, starting at different latencies. However, it is poorly understood the neural mechanism underlying the dynamic properties of IT neurons in face perception. To address this issue, we present a functional model of IT cortex, which performs face perception via an interaction between three IT layers and face recognition layer (FRL).

The model was based on the face information that is processed by three resolution maps in early visual areas. The network model of IT consists of four kinds of networks, in which the information about a whole face is combined with the information about its face parts and arrangement. The neurons of IT layers and FRL were modeled with Hodgkin-Huxley model. The synaptic connections between the four layers were made based on Hebbian learning.

We demonstrated that the spike synchrony, induced by the learning due to coincident spiking, was needed to make the functional connections between the IT layers and FRL. After the learning, the information about face parts and the whole face were combined by the synchronous firing of the IT neurons. Furthermore, "global-to-local" processing of face information could be mediated by the top-down signal between three IT layers and FRL. These results were consistent with the experiment results of the previous studies $[3,4]$. The present study provides an insight into understanding the mechanism of how the information about a whole face and its parts are processed in the IT cortex.

We presented a model of IT cortex and investigated the neural mechanism underlying the dynamic properties of IT neurons in face perception.

\section{Author details}

${ }^{1}$ Graduate School of Information Network Sciences, University of ElectroCommunications, Chofu, Tokyo, 182-8585, Japan. ${ }^{2}$ Department of Applied Physics and Chemistry, University of Electro-Communications, Chofu, Tokyo, 182-8585, Japan.

Published: 20 July 2010

\section{References}

1. Logothetis NK, Sheinberg DL: Visual object recognition. Annu Rev Neurosci 1996, 19:577-621.

2. Tanaka K: Inferotemporal cortex and object vision. Annu Rev Neurosci 1996, 19:109-139.

3. Hirabayashi T, Miyashita Y: Dynamically modulated spike correlation in monkey inferior temporal cortex depending on the feature configuration within a whole object. J Neurosci 2005, 25:10299-10307.

4. Sugase $Y$, Yamane $S$, Ueno S, Kawano K: Global and fine information coded by single neurons in the temporal visual cortex. Nature 1999, 400:869-873.

\section{doi:10.1186/1471-2202-11-S1-P21}

Cite this article as: Yamada and Kashimori: Dynamic coding of face information in inferior temporal cortex. BMC Neuroscience 201011 (Suppl 1):P21.

* Correspondence: kashi@pc.uec.ac.jp

${ }^{1}$ Graduate School of Information Network Sciences, University of Electro-

Communications, Chofu, Tokyo, 182-8585, Japan 